\title{
Advances in analytical mass spectrometry
}

\author{
Maria Careri
}

Published online: 10 January 2011

(C) Springer-Verlag 2011

This special issue of Analytical and Bioanalytical Chemistry contains a selection of 13 manuscripts dealing with the most recent advances in analytical mass spectrometry (MS). Besides updating readers on developments in analytical MS, further aims of this issue are to describe trends in both elemental and molecular MS in bioanalysis and life sciences and to provide a survey on modern applications.

The topics covered in these contributions range from matrixfree methods for laser desorption/ionization (LDI) MS to imaging MS techniques. Reviews on instrumental developments in direct coupling of liquid chromatography (LC) with MS using electron ionization, developments in protein-ligand affinity MS, and the combination of fast LC with MS for the diagnostic assay of metabolites and proteins are included. A review paper on the use of MS as a test bench for medicinal chemistry studies is presented. Trends in analytical atomic and molecular MS with a focus on biology and life sciences are also reviewed, presenting developments in coupling microfluidics with MS and in miniaturization of mass spectrometers. Matrixfree methods for LDI MS and molecular imaging MS techniques are addressed. Advances in atomic MS concern recent technological developments in MS imaging of elements, making imaging MS methods indispensable tools for obtaining information relevant to structural biology. The impressive progress shown by inductively coupled plasma (ICP) MS for high-precision trace element analysis and for speciation of elements in biological samples is also highlighted.

Pharmaceutical, medical, and bioanalytical fields have strongly pushed MS to new heights, and the significance of

Published in the special issue Advances in Analytical Mass Spectometry with Guest Editor Maria Careri.

M. Careri $(\bowtie)$

Dipartimento di Chimica Generale ed Inorganica,

Chimica Analitica, Chimica Fisica,

Università degli Studi di Parma,

Viale Usberti 17/A,

43100 Parma, Italy

e-mail: careri@unipr.it
MS techniques in those application areas is still growing rapidly. In particular, the development of MS approaches based on LDI and on ion mobility separation coupled with imaging MS brings new perspectives to biological studies. It is therefore not surprising that MS techniques applied to proteomics and metabolomics play a major role in this special issue.

Forensic analysis also benefits from technological advances in MS techniques for the purposes of separation and reliable identification of explosives and drugs.

Original papers cover an equally wide range of topics, including recent applications of imaging time-of-flight-secondary ion MS in medical research, analytical strategies for drug analysis by UPLC-MS/MS, ion mobility MS coupled with planar solid-phase microextraction for analysis of explosives, and the use of ion-exchange matrix separation coupled with ICP-MS for determination of trace element emissions for environmental monitoring.

The special issue provides both a status report on recent developments in analytical atomic and molecular MS and current trends and future perspectives in upcoming fields.

I would like to thank all the authors for their contributions to this special issue and also the editorial staff of Analytical and Bioanalytical Chemistry for fruitful cooperation.

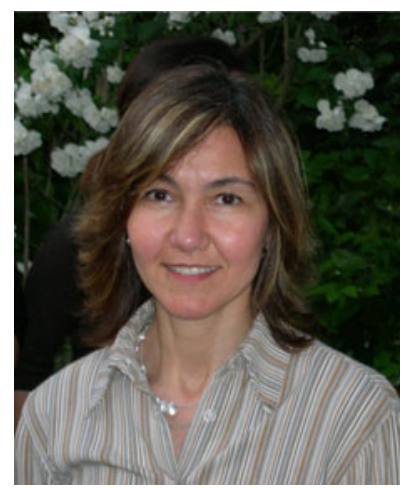

Maria Careri has been Full Professor of Analytical Chemistry at the Faculty of Sciences of Parma University since 2001. Since 2002 she has been Director of the university master course "Chemistry Laboratory Quality Systems." She is also Director of the National School of "Analytical Methodologies Based on Mass Spectrometry" of the Italian Chemical Society, which has been held in Parma since 2005. She is Past-President of the Di- 
vision of Analytical Chemistry of the Italian Chemical Society. She is a member of the editorial boards of Analytical and Bioanalytical Chemistry, Current Analytical Chemistry, and Journal of Chromatography $A$. For the last 10 years her research activities have centered on the development of novel materials for solvent-free extraction techniques for food safety and environmental monitoring and the development of innovative methods for structural and functional proteomics using mass-spectrometry-based techniques. Her current research interests include the development of novel materials for desorption electrospray ionization and matrix-assisted laser desorption/ionization mass spectrometry with special focus on the characterization of the surfaces. 